\title{
On-Line Photo-Oxidative Decomposition of Phosphorus Compounds to Orthophosphate and Its Application to Flow Injection Spectrophotometric Determinations of Total Phosphorus in River and Waste Waters
}

\author{
Keiro Higuchi*, Hiromitsu Tamanouchi** and Shoji Motomizu* \\ * Department of Chemistry, Faculty of Science, Okayama University, \\ Tsushima-naka, Okayama 700-8530, Japan \\ **Tokyo Kasei Kogyo Co. Ltd., 6-15-9, Toshima, Kita-ku, Tokyo 114-0003, Japan
}

\begin{abstract}
A simple and mild on-line photo-oxidative decomposition method for the highly sensitive flow-injection spectrophotometric determination of total phosphorus in river and waste waters was developed for the first time by using low pressure mercury lamps. Organic and inorganic phosphorus compounds in sample solutions were injected into a carrier solution, which merged with the decomposition solution containing sulfuric acid and potassium peroxodisulfate. The solution components were decomposed oxidatively to orthophosphate while flowing in a photo-reactor. After the decomposition, orthophosphate was determined spectrophotometrically based on the formation of Molybdenum Blue. The photo-reactor consisted of two low pressure mercury lamps, a poly(terafluoroethylene) (PTFE) tubing and a reflection mirror. The PTFE tubing was wound directly around the mercury lamps, which were covered with reflection mirror. The detection limit for phosphorus was about $0.001 \mathrm{mg} \mathrm{l}^{-1}$, and the sample throuphput was about 20 samples per hour for total phosphorus determination. By using the photo-reactor, most of the phosphorus compounds were decomposed completely, whereas some condensed phosphates were less susceptible to photo-oxidation. The analytical results for practical samples obtained by the proposed method showed a good correlation with those obtained by using a heat-oxidation method, which indicates that the content of the condensed phosphates difficult to be decomposed was very scarce in the practical samples.
\end{abstract}

Keywords Photo-oxidative decomposition, total phosphorus determination, flow injection analysis, Molybdenum Blue

Recently, some photochemical reactions have been applied successfully to on-line reduction and oxidation of organic and inorganic substances as a simple and a rapid pretreatment method in flow injection analysis. The photochemical reduction of nitrate to nitrite proceeds while samples are flowing in a quartz tubing coil or in a poly(tetrafluoroethylene) (PTFE) tubing coil irradiated with a low pressure mercury lamp; the method was applied to the determination of nitrate in water samples without poisonous cadmium waste. ${ }^{1,2}$ Selenium(VI) was also reduced on line to selenium(IV) in a reduction coil installed in a photo-reactor. ${ }^{3}$ On the other hand, Oguma et al. studied a photochemical oxidation of iron(II) to iron(III) in the presence of Tiron irradiated with a low pressure mercury lamp. ${ }^{4,5}$ Kodera et al. reported the determination of hydroxylamine, which was oxidized to nitrite and nitrate by irradiation of ultraviolet (UV) light. The nitrite produced was detected at $540 \mathrm{~nm}$ using a diazotization/coupling reaction. ${ }^{6}$ Dissolved organic carbons in natural and waste waters were also photo-oxidized to carbon dioxide and determined spectrophotometrically. ${ }^{7}$

In general, the decomposition of various organic and inorganic phosphorus compounds to orthophosphate, in both a batch-wise and a flow injection method, has been carried out by heating either in an autoclave ${ }^{8}$ or in a PTFE tubing reactor in the presence of potassium peroxodisulphate as a decomposition agent. A number of flow injection methods for the determination of total phosphorus have been reported. However, they involved the use of high temperatures $\left(120-160^{\circ} \mathrm{C}\right)$, increased pressures and long tubing reactors. ${ }^{9-11}$ These decomposition procedures caused some problems such as experimental danger, long analysis times or endurance of flow injection systems. Moreover, it took about $1 \mathrm{~h}$ for the decomposition temperature to reach about $150^{\circ} \mathrm{C}$ and for the heating block to cool to room temperature after analysis. In flow injection analysis, many researchers desire to develop a more rapid, simple and safe decomposition method.

Recently, some applications of UV photo-oxidation to phosphorus compounds have been reported. ${ }^{12,13}$ Mckelvie et al. determined dissolved organic phosphorus in natural waters based on photo-oxidation, where samples were flowing in PTFE tubing coil ( $0.3 \mathrm{~mm}$ i.d. $\times 3 \mathrm{~m}$ ) irradiated with a mercury lamp. ${ }^{14}$ However, the 
method required the removal of oxygen and ozone bubbles, which were formed as the products of the UV photolysis of alkaline persulfate, from the flow line before the absorbance was measured. In addition, the UV source had a power of $40 \mathrm{~W}$, so that the apparatus of the flow system became larger and much more complicated. However, the low pressure mercury lamp, which was very simple, small and safe, has never been examined so far. Moreover, the detection limit was $0.01 \mathrm{mg} \mathrm{l}^{-1}$, which was low enough to determine trace amounts of phosphorus in environmental analysis, because a method suitable for ppb levels $\left(\mathrm{ng} \mathrm{l}^{-1}\right)$ is now required.

In this paper, the spectrophotometric determination of total phosphorus based on a photo-oxidation is described. By using a low pressure mercury lamp (power consumption: $4 \mathrm{~W}$, for germicidal use) as UV irradiation source and a PTFE tubing as a reactor coil, a simple and low-cost photo-reactor for the decomposition of phosphorus compounds to orthophosphate was assembled without any complicated pieces of apparatus such as a de-bubbler. The improved spectrophotometric method based on the formation of Molybdenum Blue was applied to the determination of $\mathrm{ng} \mathrm{l}^{-1}$ level of orthophosphate formed by photo-oxidation decomposition.

\section{Experimental}

\section{Apparatus}

The flow system used in this work is shown schematically in Fig. 1. Two double-plunger micro pumps (Model 201; Tokyo Kasei Kogyo) were used for a decomposition solution, a carrier solution and a colordeveloping reagent solution. The absorbance was measured with a double-beam spectrophotometer with 10mm flow cell ( $8 \mu \mathrm{l}$ ) (Model S-3250; Tokyo Kasei

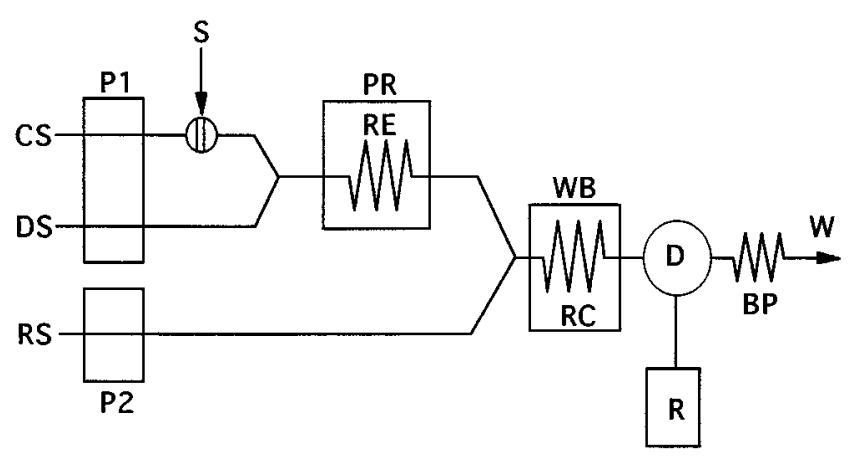

Fig. 1 Schematic FIA diagram for the determination of total phosphorus: CS, carrier solution; DS, decomposition solution; $\mathrm{RS}$, color-developing reagent solution; $\mathrm{P}_{1}$, pump (each flow rate, $\left.0.5 \mathrm{ml} \mathrm{min}^{-1}\right) ; \mathrm{P}_{2}$, pump $\left(0.5 \mathrm{ml} \mathrm{min}^{-1}\right) ; \mathrm{S}$, sample injector $(300 \mu \mathrm{l})$; PR, photo-reactor; $\mathrm{RE}$, reactor tubing $(0.5 \mathrm{~mm}$ i.d. $\times 5 \mathrm{~m})$; WB, water bath $\left(70^{\circ} \mathrm{C}\right)$; $\mathrm{RC}$, reaction coil $(0.5 \mathrm{~mm}$ i.d. $\times 3 \mathrm{~m})$; D, detector $(830 \mathrm{~nm})$; R, recorder; BP, back pressure coil $(0.25 \mathrm{~mm}$ i.d. $\times 2 \mathrm{~m})$; W, waste.
Kogyo), the peaks being recorded with a recorder (Model DR1212; Okuradenki). The sample solution $(300 \mu \mathrm{l})$ was injected via a six way injection valve into the carrier stream; it merged with the decomposition solution and the mixture flowed into a photo-reactor. The photo-reactor consisted of two low pressure mercury lamps (14 mm o.d., $134 \mathrm{~mm}$ long, $4 \mathrm{~W}$ germicidal use), a piece of PTFE tubing $(0.5 \mathrm{~mm}$ i.d., $1.58 \mathrm{~mm}$ o.d., $5 \mathrm{~m}$ long) and a reflection mirror. The PTFE tubing was wound directly around the two mercury lamps.

\section{Reagents}

All reagents used were of analytical-reagent grade and were used without further purification. Water purified with Milli-Q Labo was used for preparing all solutions and diluting them.

Decomposition solution. Sodium peroxodisulfate (5.0 g) was dissolved in 11 of $0.2 \mathrm{M}$ sulfuric acid.

Color-developing reagent solution. Ammonium molybdate tetrahydrate (5.48 g) and antimony potassium tartrate $(0.25 \mathrm{~g})$ and sulfuric acid $(67 \mathrm{ml})$ were dissolved in about $800 \mathrm{ml}$ of water, and the solution was diluted to 11 with water (Solution A). L-Ascorbic acid $(3.0 \mathrm{~g})$ and sodium dodecylsulfate $(1.0 \mathrm{~g})$ were dissolved in 11 of water (Solution B). The color-developing reagent solution was prepared by mixing equal volumes of Solutions A and B just before use. Each solution was stable for at least three months if it was stored in a refrigerator.

Carrier solution. Purfied water was used.

Standard phosphate solutions. A stock solution (1000 $\mathrm{mg}^{-1}$ ) was prepared by dissolving $4.3937 \mathrm{~g}$ of potassium dihydrogenphosphate in water to give a 1-1 solution. Working standard solutions were prepared by accurate dilution of the stock solution before use.

Model phosphorus compound solutions. Model compound solutions were prepared by dissolving the following phosphorus compounds in water; D-glucose-1phosphate dipotassium salt (G-1-P), glucose-6-phosphate dipotassium salt (G-6-P), ribose-5-phosphate barium salt (R-5-P), riboflavine phosphate sodium salt (RF-P), $\alpha$-glycerophosphoric acid disodium salt (GlyP), 1-aminoethylphosphoric acid (Amino-P), methyltriphenylphosphonium bromide (M-TP), adenosine 5'phosphoric acid (AMP), adenosine $5^{\prime}$-diphosphoric acid sodium salt (ADP), adenosine $5^{\prime}$-triphosphoric acid disodium salt (ATP). There were all available from Tokyo Kasei Kogyo. Sodium diphosphate decahydrate (Pyro-P, Kokusan-kagaku) and sodium triphosphate (Tri-P, Kanto-kagaku) were also used. Working standard solutions were prepared daily by accurate dilution of the stock model standard solutions $\left(1 \times 10^{-3}\right.$ M).

\section{Results and Discussion}

Spectrophotometric detection of orthophosphate

The FIA determination of phosphorus has been car- 
ried out mostly by the spectrophotometric method based on the formation of yellow molybdophosphate, which was produced by the reaction of orthophosphate with molybdate in an acidic medium. In order to improve the detection sensitivity and to eliminate the interference from coexisting substances, Molybdenum Blue, which was produced by the reduction of Molybdenum Yellow with ascorbic acid or tin(II) chloride, has generally been used for the determination of phosphate in environmental water samples. ${ }^{15,16}$ As a highly sensitive method, the molybdophosphate formed in an acidic medium reacted with Malachite Green to form a colored ion associate, which has been used for a sensitive spectrophotometric determination of phosphate. ${ }^{17}$ Though the spectrophotmetric method with Malachite Green was very sensitive (the molar absorptivity at $650 \mathrm{~nm}$ was about $\left.1 \times 10^{5} 1 \mathrm{~mol}^{-1} \mathrm{~cm}^{-1}\right)$, the drift of base line was sometimes occurred at high concentrations of phosphate due to the adsorption of the ion associate on a PTFE tubing and a flow cell. Therefore, in this work, the Molybdenum Blue-based spectrophotometric method with ascorbic acid, which has been widely used in environmental analysis as a standard method $^{8}$, was adopted because it had enough sensitivity for the FIA determination of total phosphorus in environmental water samples. The color-developing reagent solution used in this work was one modified for the FIA determination by referring to the previously reported method and the standard method. First, the component of the color-developing reagent solution was investigated. The addition of various kinds of anionic, cationic and nonionic surfactants to the reagent solution was examined in order to prevent the produced molybdophosphate from adsorbing on the PTFE reaction tubing coil and the flow cell. Of these, anionic surfactants such as sodium dodecyl sulfate and sodium dodecylbenzenesulfonate were the most effective; the tailings of the peaks were shortened and the linearity of the calibration graph at low concentrations of phosphorus was improved. This is probably because the anionic surfactants will adsorb on the wall of the PTFE tubing and the flow cell, which can interfere with the adsorption of anionic Molybdenum Blue on the wall.

The effect of the temperature of the reaction coil on the color-developing reaction was examined by varing the temperature of the water bath from room temperature $\left(25^{\circ} \mathrm{C}\right)$ to $90^{\circ} \mathrm{C}$ to improve the detection sensitivity and the linearity of a calibration graph. The peak heights for orthophosphate increased with an increase in temperature, and reached almost to the maximum at about $70^{\circ} \mathrm{C}$, where the linearity of the calibration graph was very good even at low concentration ranges of orthophosphate. Further experiments were carried out at $70^{\circ} \mathrm{C}$ of the water bath for the reaction coil.

\section{Assembly of photo-reactor}

By using the low-pressure mercury lamps (power consumption of $4 \mathrm{~W}$ for germidical use), the decomposition of the phosphorus compounds was investigated by varying the components of the decomposition solution. As a result, it was found that the low-pressure mercury lamps (power consumption of $30-60 \mathrm{~W}$ ) has been very useful for the decomposition of organic phosphorus compounds. Such lamps, however, are in general very expensive and a large amount of heat is generated. Therefore, the decomposition apparatus becomes more complicated and larger. In this work, we aimed at developing a simple, low-cost and easy-tohandle apparatus for the decomposition of organic and inorganic phosphorus compounds generally existing in environmental water samples. Motomizu et al. already reported the photo-oxidative decomposition of hydroxylamine $^{6}$, in which a low pressure mercury lamp was used. In this work, we also investigated the low pressure mercury lamp for the decomposition of various phosphorus compounds to orthophosphate. By using the low pressure lamps, it was possible to miniaturize and to simplify the FIA system, and to improve the safety of the operation. In the previous study ${ }^{2}$, PTFE and polyethylene (PE) tubing were examined. The result obtained showed that PE tubing was less effective than PTFE tubing as a reactor tubing because of less transparency of UV-light. Therefore PTFE tubing was selected and examined. By using PTFE tubing, which was easily coiled around the mercury lamps, the reduction of $\mathrm{NO}_{3}{ }^{-}$to $\mathrm{NO}_{2}{ }^{-}$was enhanced. The reduction efficiency reached about $70-84 \%$, which was higher compared with that of a quartz coil reported by Takeda et al. ${ }^{1}$

The effect of the inner diameter of PTFE tubing on the decomposition rate was examined with $1.0 \mathrm{~mm}$ i.d., $0.8 \mathrm{~mm}$ i.d. and $0.5 \mathrm{~mm}$ i.d. There was no difference in the decomposition rate; therefore, the tubing of $0.5 \mathrm{~mm}$ i.d. was adopted to achieve the lower dispersion of sample zone. Effect of reactor-tubing length on the decomposition rate was examined by varying the length of tubing $(0.5 \mathrm{~mm}$ i.d., $1.58 \mathrm{~mm}$ o.d.) in photo-reactor. The tubing was wound around the mercury lamps and covered with reflection mirror. A 3-m tubing could be wound around one lamp, and the maximum decomposition rate was obtained by using $5-\mathrm{m}$ tubing wound around two lamps. In the present system, it was unnecessary to install a de-bubbler, because the heat generation was very small, and the formations of oxygen and ozone bubbles were not observed when the back pressure tubing $(0.25 \mathrm{~mm}$ i.d. $\times 2 \mathrm{~m})$ was attached after the detector.

The effect of flow rates at identical rate was examined by varying the flow rate from $0.2 \mathrm{ml} \mathrm{min}^{-1}$ to 1.0

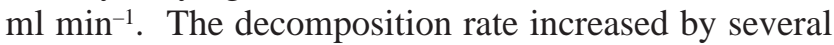
percentages for the condensed phosphorus compounds with decreasing the flow rates. In this work, $0.5 \mathrm{ml}$ $\mathrm{min}^{-1}$ was adopted as a compromise of an analytical time and a decomposition rate.

\section{Effect of UV irradiation on the photo-oxidation of phosphorus compounds \\ The effect of UV irradiation on the photo-oxidation}


was investigated using several series of model phosphorus compounds. Figure 2 shows the flow signals for $3 \times 10^{-5} \mathrm{M}$ of various phosphorus compounds with the UV lamps on or off. When the UV lamps were turned on, it was clear by comparing the peak height of the model phosphorus compounds with that of orthophosphate used as a standard material that most of the model compounds were oxidized almost quantitatively, except for condensed phophorus compounds (ADP, ATP, pyrophosphate and triphosphate), and the oxidation became less effective when the lamps were turned off. Glucose-, ribose-, riboflavine- and glycerophosphate, which contained an ester bond, were found to be more photo-oxidative with peroxodisulfate in the presence of an acid. Methyltriphenylphosphonium ion possessing a C-P bond, which was known to be quite resistant to hydrolysis and oxidation, also could be photo-oxidized under the proposed mild conditions. However, the series of phosphorus compounds possessing a condensed phosphate bond, such as ADP, ATP,

(a)

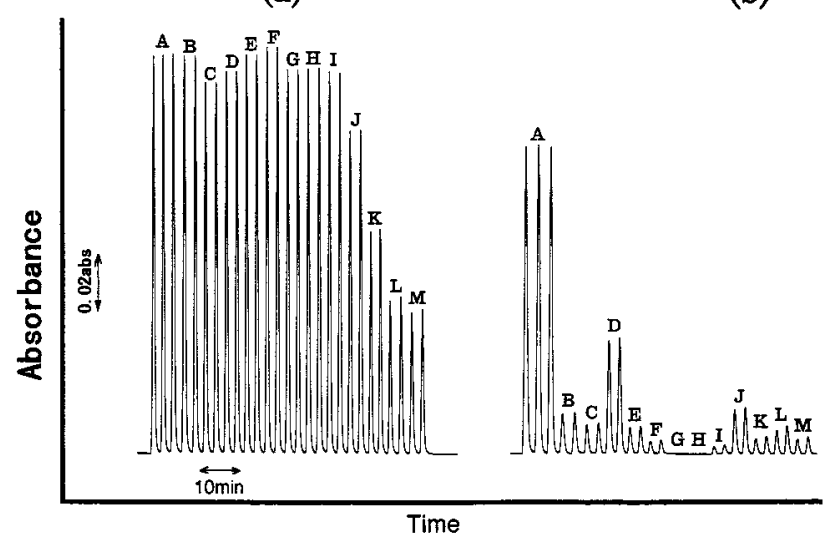

Fig. 2 Effect of UV irradiation on the photo-oxidation of phosphorus compounds: (a) lamp on; (b) lamp off. A, orthophosphate; B, G-1-P; C, G-6-P; D, R-5-P; E, RF-P; F, Gly-P; G, Amino-P; H, M-TP; I, AMP; J, ADP; K, ATP; L, Pyro-P; M, Tri-P. Decomposition solution: $0.5 \%$ of potassium peroxodisulfate in $0.2 \mathrm{M}$ sulfuric acid. pyrophosphate and tripolyphosphate, were difficult to be decomposed by the low pressure UV irradiation. From these results, it was found that the UV irradiation examined in this work was very effective in the oxidative decomposition of organic phosphorus compounds, but less effective in the hydrolysis of the condensed phosphate. Therefore, the components of the decomposition solution were further examined.

\section{Components of decomposition solution}

The effect of the concentration of potassium peroxodisulfate in the decomposition solution was examined. The concentration of potassium peroxodisulfate in the decomposition solution containing 0.2 M sulfuric acid on the rate of decomposition of condensed phosphorus compounds to orthophosphate was investigated over the ranges from 0 to $4 \%(\mathrm{w} / \mathrm{v})$. The conversion rate of the series of model compounds was maximum and constant at the concentrations of more than $0.2 \%$. In further experiments, a concentration of potassium peroxodisulfate of $0.5 \%$ was chosen for safety.

The effect of the concentration of sulfuric acid in the decomposition solution was examined. Figure 3 shows the effect of the concentration of sulfuric acid in the decomposition solution containing $0.5 \%$ of potassium peroxodisulfate on the conversion rate. The conversion rates of the most of model compounds were about $100 \%$ at $0.2 \mathrm{M}$ sulfuric acid, except for the condensed phosphorus compounds. However, the conversion rate of each condensed phosphorus compound increased with increasing the concentration of sulfuric acid, though it was difficult to convert the condensed phosphate completely to orthophsphate. It was found that the condensed phosphate compounds could be converted to orthophosphate almost completely at temperatures higher than $100^{\circ} \mathrm{C}$. In this work, special heating was not adopted because the amounts of condenced phosphate are usually very scarce in environmental water samples compared with the amount of orthophosphate and organic phosphorus compounds. Considering the sensitivity of detection and simplicity of the preparation of solution, we chose $0.2 \mathrm{M}$ sulfuric acid. (a)

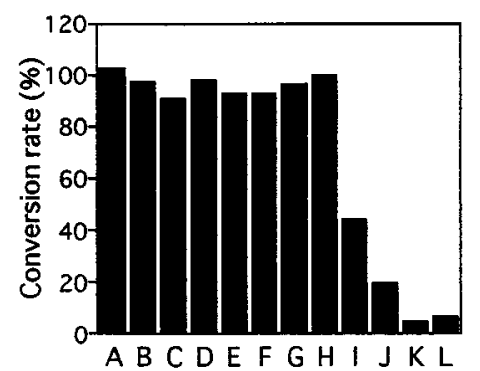

(b)

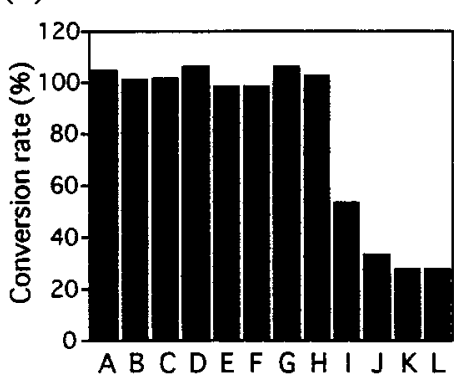

(c)

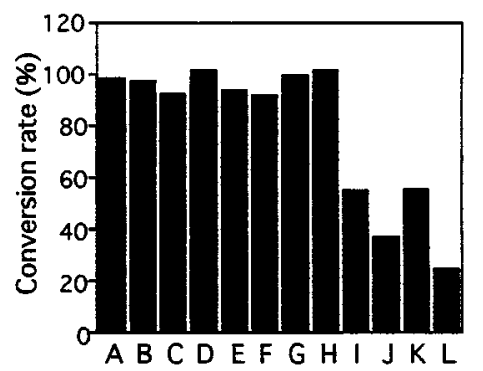

Fig. 3 Effect of concentration of sulfuric acid in the decomposition solution. $\mathrm{H}_{2} \mathrm{SO}_{4}$ : (a), $0.2 \mathrm{M}$; (b), 1.0 M; (c), 2.0 M. A, orthophosphate; B, G-1-P; C, G-6-P; D, R-5-P; E, RF-P; F, Gly-P; G, Amino-P; H, M-TP; I, AMP; J, ADP; K, ATP; L, Pyro-P; M, Tri-P. 
Calibration graph and detection limit for standard phosphate solution

Calibration graphs for orthophosphate were obtained by using the proposed system and experimental conditions. Figure 4 shows the flow signals for the calibration graphs of orthophosphate. The reproducibility of the peak height was very good, and the calibration graphs were linear over the ranges of 0 to $0.1 \mathrm{mg} \mathrm{l}^{-1}$ ( 0 $-100 \mathrm{ppb})$ and 0 to $1.0 \mathrm{mg} \mathrm{l}^{-1}(0-1.0 \mathrm{ppm})$ of $\mathrm{P}_{-} \mathrm{PO}_{4}{ }^{3-}$. The detection limit corresponding to the signal to noise ratio $(S / N)$ of 3 was about $0.001 \mathrm{mg} \mathrm{l}^{-1}(1 \mathrm{ppb})$ of $\mathrm{P}$ $\mathrm{PO}_{4}{ }^{3-}$, and the sample throughput was about 20 samples per hour for the total phosphorus determination including the photo-oxidation step.

\section{Application to the determination of total phosphorus in river and waste waters}

The proposed method was applied to the determination of total phosphorus in river and waste waters. Table 1 shows the results of the repeatability tests and the recovery tests obtained by using the proposed photo-oxidation/FIA method. The relative standard deviations of ten injections were in the ranges of $0.13 \%$ to $2.25 \%$. The recovery of orthophosphorus was found

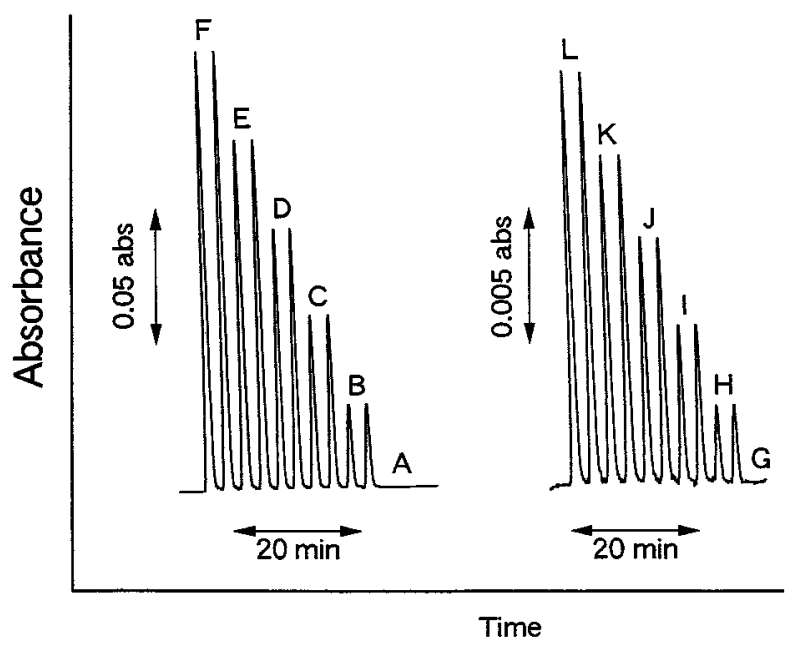

Fig. 4 Flow signals for the calibration graph of orthophosphate. $\left[\mathrm{P}_{-} \mathrm{PO}_{4}{ }^{3-}\right] / \mu \mathrm{g} \mathrm{ml}^{-1}: \mathrm{A}, 0 ; \mathrm{B}, 0.2 ; \mathrm{C}, 0.4 ; \mathrm{D}, 0.6 ; \mathrm{E}, 0.8$; F, 1.0; G, 0; H, 0.02; I, 0.04; J, 0.06; K, 0.08; L, 0.10. to be $99 \%$ to $102 \%$ with the concentration ranges of 0.024 to $3.03 \mathrm{mg} \mathrm{l}^{-1}$. The results indicate that both the repeatability and the recovery of the determination of total phosphorus are enough for the applicability of the proposed method to environmental water samples.

The heat-oxidation method was investigated in order to compare it with the proposed photo-oxidation method. The heat-oxidation system consisted of a PTFE tubing $(0.5 \mathrm{~mm}$ i.d. $\times 10 \mathrm{~m})$ wound around the aluminum rod heated at $135^{\circ} \mathrm{C}$, instead of the proposed photo-reactor shown in Fig.1. In the heat-oxidation method, the conditions for the color-developing reaction were the same as in the Molybdenum Blue method used in this work and the decomposition reaction was the same as in the proposed photo-oxidation system. The conversion rates of the series of model compounds obtained by using the flow system of the heat-oxidation are summarized in Table 2. It is evident that even the compounds possessing the condensed phosphate bond, such as ADP, ATP and triphosphate which could not be decomposed completely by using the proposed photoreactor, could be decomposed to orthophosphate almost completely.

Various practical samples of river and waste waters were analyzed by both the proposed photo-oxidation/ FIA method and the heat-oxidation/FIA method. Figure 5 shows the correlation between two methods. It can be seen from Fig. 5 that there are good correlations over the entire concentration ranges of 0.006 to 5.84 $\mathrm{mg}^{-1}$ of phosphorus with a regression equation as follows: $y=0.9797 x-0.0325,(r=0.9986, n=10)$, where $y$ and $x$ are the phosphorus contents obtained by the proposed photo-oxidation/FIA method and the heat-oxidation/FIA method, respectively. These results demonstrate that the proposed photo-oxidation method is useful for the determination of total phosphorus in practical river and waste waters.

In practical analysis, the proposed photo-oxidation/ FIA method seemed to be superior to the heat-oxidation/ FIA method for the reasons that it is unnecessary to heat the decomposition tubing with use of a heating reactor, and the dangers such as burst of tubing and over heating of apparatus can be avoided in photo-oxidation method with low-pressure mercury lamps.

Table 1 Repeatability and recovery tests for the determination of total phosphorus in river and waste waters

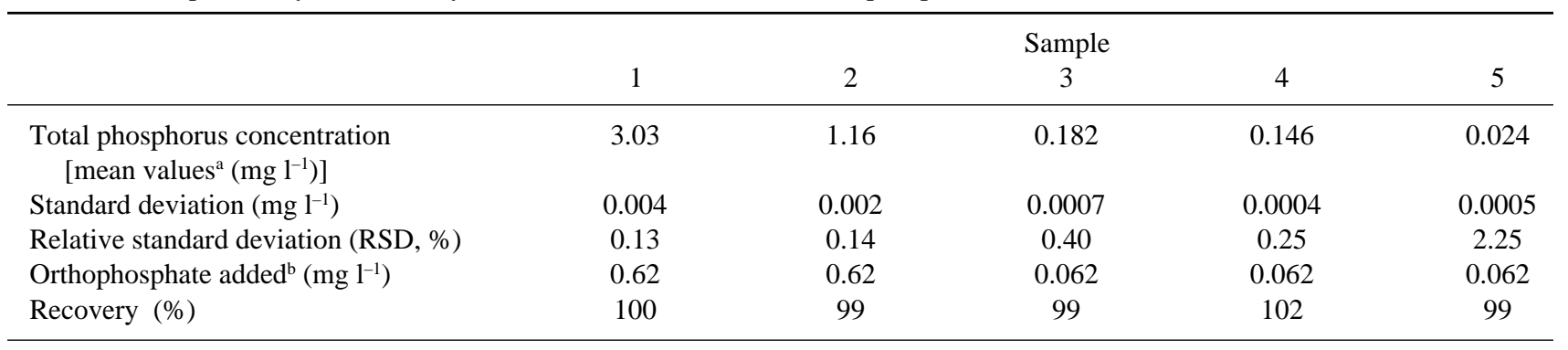

a. Each sample was analyzed by the proposed FIA method, and 10 measurements were carried out. b. Each sample was diluted twice with purified water after the addition of known amounts of standard orthophosphate solution. 
Table 2 Conversion rate for various phosphorus compounds by a heat-oxidation method

\begin{tabular}{lccc}
\hline Compound & $\begin{array}{c}\text { Conversion } \\
\text { rate, } \%\end{array}$ & Compound & $\begin{array}{c}\text { Conversion } \\
\text { rate, \% }\end{array}$ \\
\hline G-1-P & 98.8 & M-TP & 95.0 \\
G-6-P & 98.9 & AMP & 97.6 \\
R-5-P & 93.0 & ADP & 89.2 \\
RF-P & 99.0 & ATP & 87.5 \\
Gly-P & 99.6 & Pyro-P & 98.1 \\
Amino-P & 93.8 & Tri-P & 98.1 \\
\hline
\end{tabular}

Phosphorus: $3 \times 10^{-5} \mathrm{M}$.

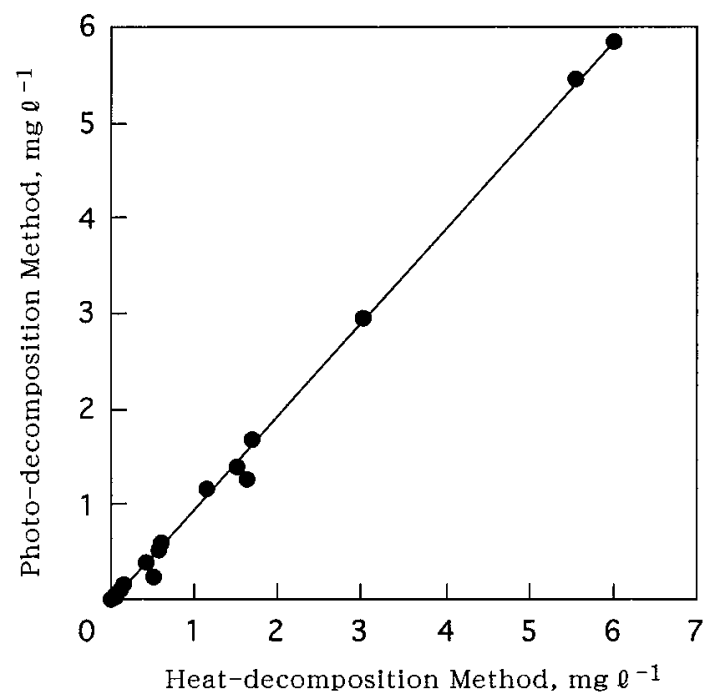

Fig. 5 Correlation between the results obtained by the proposed photo-oxidation method and the heat-oxidation method for the determination of total phosphorus in practical river and waste waters. The line can be represented by the following equation: $y=0.9797 x-0.0325(r=0.9986, n=10)$, where $y$ and $x$ are the phosphorus contents obtained by the proposed photo-oxidation/FIA method and the heat-oxidation/FI method, respectively.

Moreover, the proposed photo-reactor can be easily miniaturized because of using only two low-pressure mercury lamps and a small-sized power source. The method proposed here is very useful for practical analyses because of the mild conditions, the improvement of safety, the simplicity of flow system, high sensitivity and reproducibility.

\section{References}

1. K. Takeda and K. Fujiwara, Anal. Chim. Acta, 276, 25 (1993).

2. S. Motomizu and M. Sanada, Anal. Chim. Acta, 308, 406 (1995).

3. M. J. Ahmed, C. D. Stalikas, P. G. Veltsistas, S. M. Tzouwara-Karayanni and M. I. Karayannis, Analyst [London], 122, 221 (1997).

4. R. Kuroda, T. Nara and K. Oguma, Analyst [London], 113, 1557 (1988).

5. K. Oguma, S. Kozuka, K. Kitada and R. Kuroda, Fresenius' J. Anal. Chem., 341, 545 (1991).

6. T. Kodera, M. Oshima and S. Motomizu, J. Flow Injection Anal., 13, 25 (1996).

7. R. T. Edwards, I. D. Mckelvie, P. C. Ferrett, B. T. Hart and J. B. Bapat, Anal. Chim. Acta, 261, 287 (1992).

8. Japanese Industrial Standard K0102 (1993).

9. T. Korenaga and K. Okada, Bunseki Kagaku, 33, 683 (1984).

10. M. Goto, M. Nishimura, T. Tominaga and D. Ishii, Bunseki Kagaku, 37, 52 (1988).

11. M. Aoyagi, Y. Yasumasa and A. Nishida, Anal. Chim. Acta, 214, 229 (1988).

12. A. Henriksen, Analyst [London], 95, 601 (1970).

13. J. T. H. Goossen and J. G. Kloosterber, Anal. Chem., 501, 707 (1978).

14. I. D. Mckelvie, B. T. Hart, T. J. Cardwell and R. W. Cattrall, Analyst [London], 114, 1459 (1989).

15. J. Ruzicka and E. H. Hansen, Anal. Chim. Acta, 78, 145 (1975).

16. P. R. Freeman, I. D. Mckelvie and B. T. Hart, Anal. Chim. Acta, 234, 409 (1990).

17. S. Motomizu, T. Wakimoto and K.Tôei, Talanta, 30, 333 (1983).

(Received April 15, 1998)

(Accepted July 6, 1998) 